\title{
Influence of Long-Term Feeding Antibiotics on the Gut Health of Zebrafish
}

\author{
Li Zhou, Samwel Mchele Limbu, ${ }^{1,2}$ Fang Qiao, Zhen-Yu Du, and Meiling Zhang ${ }^{1}$
}

\begin{abstract}
The use of antibiotics for anti-infection and growth promotion has caused the overuse of antibiotics in aquaculture. However, the benefit or risk of the long-term use of antibiotics on fish growth or health has not been fully addressed. In the present study, zebrafish were fed with sulfamethoxazole (SMX) or oxytetracycline (OTC) at the therapeutic concentrations (100 and $80 \mathrm{mg} / \mathrm{kg}$ body weight per day, respectively) for 6 weeks to mimic the long-term use of antibiotics. The digestive enzyme activities were higher in both antibiotic treatments, and higher oxygen consumption rate was found in OTC treated group. As a result, SMX increased the weight gain of zebrafish, and OTC treatment did not show significant prompting effect on growth. The mortality was higher in SMX or OTC treated group on 2nd-4th day after exposure to Aeromonas hydrophila. Lower alkaline phosphatase (AKP) and acid phosphatase (ACP) activities were found in OTC treated group, while higher malondialdehyde (MDA) content was found in the intestine of both SMX and OTC treated zebrafish. Furthermore, feeding OTC decreased the intestinal microbial richness. This study revealed that long-term use of legal aquaculture concentrations of antibiotics caused systemic adverse effects on fish gut health; stringent policy for use of antibiotics in fish is urgent.
\end{abstract}

Keywords: antibiotics, growth, immune function, intestinal microbiota, intestinal health

\section{Introduction}

C HINA IS CURRENTLY the largest aquaculture producer and exporter, contributing $61.7 \%$ of the global aquaculture production. ${ }^{1}$ With the rapid development of aquaculture industry, intensive culture has been more and more popular, but uneaten feeds and wastes excreted from fish deteriorate water quality, which cause the prevalence of diseases. ${ }^{2}$ Increased fish stocking density, which may cause crowding or hypoxic stress, and the lack of sanitary barriers between farming sites increase the potential risk for the spread of infection. ${ }^{2-4}$

In Chinese cultured aquatic species, more than 200 diseases have been identified with bacterial infections. These infections are estimated to account for $15 \%-20 \%$ of annual total production, which implies an economic loss of 5-7 billion Chinese Yuan (about 750-1000 million US dollars). ${ }^{5}$ How to control bacterial infections is one of the most important challenges that farmers need to solve. Antibiotics are natural or synthetic drugs with the capacity to kill or inhibit the growth of bacteria, and it has been thought that with the development of intensive culture, feeding antibiotics to fish is necessary in aquaculture. ${ }^{2,6}$
Except for anti-infection, some antibiotics are also used as feed supplements to promote growth of livestock, ${ }^{7}$ which also drive the misuse or overuse of antibiotics in aquaculture. ${ }^{8}$ In China, antibiotics as growth promoters are widely used in aquafeeds, ${ }^{9}$ and oral administration of antibiotics by mixing the drugs with the fish feeds is the most common application method. ${ }^{10}$ Antibiotic treatments in aquaculture cause antibiotic resistant bacteria or genes and side effects on fish health. ${ }^{11,12}$ Many studies have detected the concentrations of antibiotic residues and occurrence of specific antibiotic resistant bacteria and genes in fish or environments, ${ }^{11,13-15}$ but the influence of antibiotics on fish health should also be taken into account.

Intestine is the important organ for preventing the entrance of pathogens and metabolizing drugs. ${ }^{16,17}$ Furthermore, intestinal microbiota also plays important roles in drug metabolism and immune regulation. ${ }^{18,19}$ Impaired intestinal barrier or function and disturbed intestinal microbiota could increase the disease susceptibility in fish, so gut health has received more and more attention when we evaluate the growth condition of fish. ${ }^{20,21}$

Sulfamethoxazole (SMX) and oxytetracycline (OTC) are two common antibiotics used in human and veterinary

\footnotetext{
${ }^{1}$ Laboratory of Aquaculture Nutrition and Environmental Health (LANEH), School of Life Sciences, East China Normal University, Shanghai, China.

${ }^{2}$ Department of Aquatic Sciences and Fisheries Technology, University of Dar es Salaam, Dar es Salaam, Tanzania.
} 
medicine $e^{22,23}$ and are the permitted antibiotics to be used in aquaculture for the treatment of systemic bacterial infections in many countries. ${ }^{12}$ In China, the recommended dose is 50$100 \mathrm{mg} \mathrm{SMX} \mathrm{kg}^{-1}$ body weight per day for 5-7 days and 50$80 \mathrm{mg} \mathrm{OTC} \mathrm{kg}^{-1}$ body weight per day for 5-10 days. ${ }^{24}$ OTC is a broad-spectrum antibiotic, which enters the bacterial cells and binds to ribosome to prevent binding of aminoacyl transfer RNA (tRNA). ${ }^{25}$ SMX, which is a structural analog of para-aminobenzoic acid (PABA), interferes with the normal bacterial synthesis of folic acid.

Although these two antibiotics are thought to have minimal side effects, they still impaired the antioxidative system or suppressed the immune system in liver, heart, kidney, or spleen of rainbow trout or carp. ${ }^{12,26,27}$ However, the influence of the long-term use of these two antibiotics at the therapeutic level on the gut health of fish remains unclear.

Zebrafish was selected as a model species and orally treated with SMX and OTC at the therapeutic level for 6 weeks to mimic the overuse of antibiotics in this study. Weight gain of fish was measured during the experiment and at the end of the experiment and then fish were challenged with pathogenic bacteria, Aeromonas hydrophila, to evaluate the anti-infection ability of zebrafish. Considering the important role of intestine and microbiota in fish health, the digestive enzymes, immunology-related enzymes, inflammatory factors, and intestinal bacterial composition were evaluated to show the risk and benefit of the long-term use of antibiotics on fish growth and health.

\section{Materials and Methods}

All experiments were conducted under the Guidance of the Care and Use of Laboratory Animals in China. This research was approved by the Committee on the Ethics of Animal Experiments of East China Normal University.

\section{Experimental animals and antibiotic exposure}

Seven hundred adult zebrafish $(0.1 \mathrm{~g}$ each) were purchased from Yuyi tropical fish farm (Shanghai, China). Fish were acclimatized for 2 weeks in $100 \times 45 \times 45 \mathrm{~cm}$ tanks with aerated dechlorinated tap water and a natural photoperiod of $14 \mathrm{~h}$ light and $10 \mathrm{~h}$ dark before the experiment. During the acclimatization, fish were fed with a commercial diet (Shengsuo Co., Shandong, China) twice per day. The water temperature and $\mathrm{pH}$ were kept at $26^{\circ} \mathrm{C} \pm 2^{\circ} \mathrm{C}$ and $\mathrm{pH}$ 6.8-7.5, respectively.

SMX (CAS: 723-46-6) and OTC (CAS: 615 3-64-6) were purchased from Yuanmu Biotechnology Co., Ltd. (Shanghai, China). According to Chinese guidelines for the use of fishery drugs NY 5071-2002, ${ }^{24}$ the concentrations of SMX and OTC used in the present study were at 100 and $80 \mathrm{mg} / \mathrm{kg}$ weight per day, respectively. SMX and OTC were weighted and mixed with the feed components at a concentration of $5 \mathrm{~g} / \mathrm{kg}$ diet and $4 \mathrm{~g} / \mathrm{kg}$ diet, respectively.

After 2 weeks of acclimatization, 600 zebrafish were randomly divided into three groups as follows: control, SMX diet, and OTC diet, and each group has triplicate tanks. The tank size is $60 \times 45 \times 45 \mathrm{~cm}$. During the experiment, fish were fed with a basic diet without antibiotics at a ratio of $2 \%$ of body weight, twice per day (Supplementary Table S1; Supplementary Data are available online at www.liebertpub. com/zeb). The weight of the fish in each tank was recorded every 2 weeks during the experiment, and the quantity of feed was adjusted correspondingly. All treatments were performed in sterile tanks, and half of the water from each tank was replaced every 2 days. The duration of the experiment lasted for 6 weeks. At the end of the experiment, fish were captured and their final weight was determined.

\section{Determination of oxygen consumption rate}

The metabolic rate was evaluated by examining the oxygen consumption rate (OCR) as previously described. ${ }^{28}$

\section{Bacterial challenge}

At the end of the experiment, A. hydrophila was used to challenge the zebrafish. The bacterial challenge was conducted in three treatments (control, SMX diet, and OTC diet), and each treatment had 25 fish in three replicates. A single colony of $A$. hydrophila was grown in Luria-Bertani (LB) liquid medium at $37^{\circ} \mathrm{C}$ with shaking at $200 \mathrm{rpm}$ for $14 \mathrm{~h}$. The overnight culture was then inoculated into $100 \mathrm{~mL}$ of fresh LB medium (1:100). The cultured bacterial cells were centrifuged and resuspended in phosphate buffered saline ( $\mathrm{pH}$ 7.4). Zebrafish were immersed in water containing bacteria with a final concentration of $5 \times 10^{8}$ colony forming unit $/ \mathrm{mL}$ for 6 days. The water and bacteria were changed every 2 days, and mortality was recorded every day.

\section{Biochemical assays}

The whole guts of three fish were pooled together, weighed, and homogenized with 9 volumes (v/w) of $0.8 \%$ physiological saline. Then, the homogenate was centrifuged at $2500 \mathrm{~g}$ at $4^{\circ} \mathrm{C}$ for $10 \mathrm{~min}$, and the supernatant was collected for biochemical assays according to the manufacturer's instructions. Amylase (AMS), lipase (LPS), and malondialdehyde (MDA) were measured using an Enzyme-linked Immunosorbent Assay Kit (Hengyuan, Shanghai, China). Superoxide dismutase (SOD), peroxidase (POD), reduced glutathione $(\mathrm{GSH})$, acid phosphatase (ACP), and alkaline phosphatase (AKP) were measured using related commercial assay kits (Nanjing Jiancheng Institute). Results were recorded on a microplate reader (Epoch; BioTek).

\section{Quantitative real-time polymerase chain reaction}

Total RNA was extracted from zebrafish gut using TRIpure Reagent (Aidlab). RNA having an A260/A280 absorbance ratio of 1.8-2.0 and an A260/A230 ratio >2.0 were used for subsequent analysis. Electrophoresis was also used to assess the RNA quality by visualizing the $28 \mathrm{~S} / 18 \mathrm{~S}$ ribosomal RNA (rRNA) ratio on a $1 \%$ agarose gel. RNA quantity was measured using NanoDrop 2000 Spectrophotometer (Thermo Scientific). The complementary DNA (cDNA) was synthesized using $1 \mu \mathrm{g}$ total RNA as the template by a PrimeScript $^{\text {TM }}$ RT Reagent Kit (Takara, Dalian, China) according to the manufacturer's instructions.

Real-time polymerase chain reaction (PCR) was conducted in the CFX Connect Real-Time System (Bio-Rad). Expression level of genes related to nutrient transportation, including $\mathrm{Na}^{+} /$ $K^{+}$-ATPase (sodium-potassium adenosine triphosphatase), Glut2 (glucose transporter 2), SGLT1 (sodium-glucose cotransporter 1), and $y^{+} L A T 1$ (light chain- ${ }^{+} \mathrm{L}$ amino acid transporter-1), was detected by real-time PCR. Genes related 
to pro-inflammatory cytokines such as tumor necrosis factor alpha $(T N F-\alpha)$, interleukin-1 $(I L-1)$, and anti-inflammatory cytokines, including transforming growth factor-beta $(T G F-\beta)$, were also detected in this study. Elongation factor- $1 \alpha(E F-1 \alpha)$ was used as the internal control to normalize the data. The primers for $E F-1 \alpha$ and target genes for quantitative PCR (qPCR) are listed in Supplementary Table S2.

The mixture for qPCR contained $10 \mu \mathrm{L}$ of $2 \times \mathrm{SYBR}$ qPCR Mixture (Aidlab), $100 \mathrm{ng}$ cDNA, $300 \mathrm{nM}$ of qPCR primers, and $6.4 \mu \mathrm{L}$ nuclease-free water. The program for the $\mathrm{qPCR}$ was $94^{\circ} \mathrm{C}$ for $3 \mathrm{~min}$ and 40 cycles at $94^{\circ} \mathrm{C}$ for $6 \mathrm{~s}$ and $60^{\circ} \mathrm{C}$ for $30 \mathrm{~s}$. The melting curves for the amplified products were generated to ensure the specificity of assays at the end of each PCR. Gene expression quantification was calculated using the comparative $\Delta \Delta \mathrm{CT}$ method, and the gene expression level in the control group was used as the comparator. All assays were performed in triplicate.

\section{Bacterial genomic DNA extraction}

Considering the limited amount of intestinal content and interindividual variation of intestinal microbiota, the intestinal contents of three fish from each tank were mixed as one sample. The intestinal contents were collected in sterile tube and immediately stored in liquid nitrogen, then transferred to fridges and kept frozen at $-80^{\circ} \mathrm{C}$ until DNA extraction. Microbial DNA was extracted according to the protocol described by the E.Z.N.A Soil DNA Kit (Omega Bio-Tek, Norcross, GA). DNA quality was assessed by gel electrophoresis and spectrophotometry (optical density 260/280). DNA yield was measured with NanoDrop 2000 Spectrophotometer (Thermo Scientific). Extracted DNA was stored at $-20^{\circ} \mathrm{C}$.

\section{Illumina high-throughput sequencing of barcoded $16 S$ rRNA genes}

The V3-V4 region of the bacteria 16S rRNA gene was amplified by PCR using primers 338F: 5'-ACTCCTACGGG AGGCAGCA-3' and 806R: 5'-GGACTACHVGGGTWT CTAAT-3'. Unique eight-base barcodes were added to each primer to distinguish the different PCR products. PCRs were performed in a $20 \mu \mathrm{L}$ mixture containing $4 \mu \mathrm{L}$ of $5 \times$ Fast Pfu Buffer, $250 \mathrm{nM}$ dNTPs, $200 \mathrm{nM}$ of each primer, $1 \mathrm{U}$ of Fast Pfu Polymerase (TransGen), and $10 \mathrm{ng}$ of template DNA. The PCR conditions were as follows: $95^{\circ} \mathrm{C}$ for $3 \mathrm{~min}$ to allow DNA denaturation, with the amplification lasting 23 cycles $\left(95^{\circ} \mathrm{C}\right.$ for $30 \mathrm{~s}, 55^{\circ} \mathrm{C}$ for $30 \mathrm{~s}$, and $72^{\circ} \mathrm{C}$ for $45 \mathrm{~s}$ ), and the final extension period lasted for $10 \mathrm{~min}$ at $72^{\circ} \mathrm{C}$. Purified PCR products were subjected to Illumina MiSeq PE300 platform, generating paired-end reads (Majorbio Bio-Pharm Technology, Co., Ltd., Shanghai, China). The sequences obtained has been submitted to GenBank with the accession number SRP111618.

\section{Bioinformatics analysis}

The raw pair-end readings were subjected to a qualitycontrol procedure using QIIME (version 1.17). The qualified reads were clustered to generate operational taxonomic units (OTUs) at the 97\% similarity level using UPARSE (version 7.1). Chimeric sequences were identified and removed using UCHIME (version 4.1). The phylogenetic affiliation of each $16 \mathrm{~S}$ rRNA gene sequence was analyzed using RDP Classifier against the SILVA 16 S rRNA database using a confidence threshold of $70 \%$. Taxonomic richness and diversity estimators were determined in Mothur.

$\mathrm{ACE}$ and Chao were used to reflect community richness, and diversity was assessed using Shannon and Simpson indices. All these indices were estimated based on OTUs abundance matrices. Principal component analysis (PCA) and heat-map analysis were performed under a MATLAB R2016a environment. Twenty-six OTUs were selected for heatmap analysis according to: (1) the abundances of these OTUs were significantly different between the control and antibiotic treated group based on Student's $t$-test and (2) the abundances of these OTUs were higher than $0.1 \%$ at least in one treatment.

\section{Statistical analyses}

All results are presented as mean \pm standard error. To detect the influence of antibiotics on fish health, Student's $t$-test was used to independently compare antibiotic treated group with the control group. All statistical analyses were conducted using SPSS software (version 18.0, Chicago, IL). A value of $p<0.05$ was regarded as statistical significance.

\section{Results}

Effects of antibiotic treatment on weight gain

and mortality rate after bacterial challenge of zebrafish

During the experiment, the body weight of three groups was detected, and the results showed that antibiotic treated groups had higher weight gain than the control group, but no significant difference has been detected on 14th and 28th day. At the end of the experiment, SMX-diet group showed significantly higher final body weight than the control group $(p<0.05)$. OTC-diet group also had relatively higher final body weight than the control group, but no significant difference has been detected ( $p>0.05$, Fig. 1A). The whole fish OCR was detected in all three treatments, and the results showed that OCR significantly increased in the OTC treated group ( $p<0.05$, Fig. 1B).

At the end of the experiment, zebrafish were challenged with A. hydrophila, and the mortality rate was monitored for another 6 days. The mortality rate began to show significant difference between the control and OTC treated group from the 2 nd to 3 rd day after the infection (Fig. 1C, $p<0.05$ ), while SMX-diet group showed significantly higher mortality than the control group on the 4th day after the infection $(p<0.05)$. Four days later, no significant difference of mortality has been found between the control and antibiotic treatment. These results indicated that the killing rate of $A$. hydrophila increased in antibiotic treated groups.

\section{Influence of antibiotic treatments on the activities of intestinal digestive enzymes and the expression of nutrient transporter genes}

Considering the higher body weight in SMX group, we examined the digestive enzymes and nutrient transporter transcript levels in the intestine. The results indicated that activities of AMS and LPS in SMX or OTC treated group were significantly higher than those in control group ( $p<0.05$, Fig. 2A, B). The expression level of transporter genes for sodium and potassium was higher in OTC treated group ( $p<0.05$, Fig. 2C). The expression levels of Glut2 and SGLT1, which are reasonable for glucose movement across cell membrane, were also 

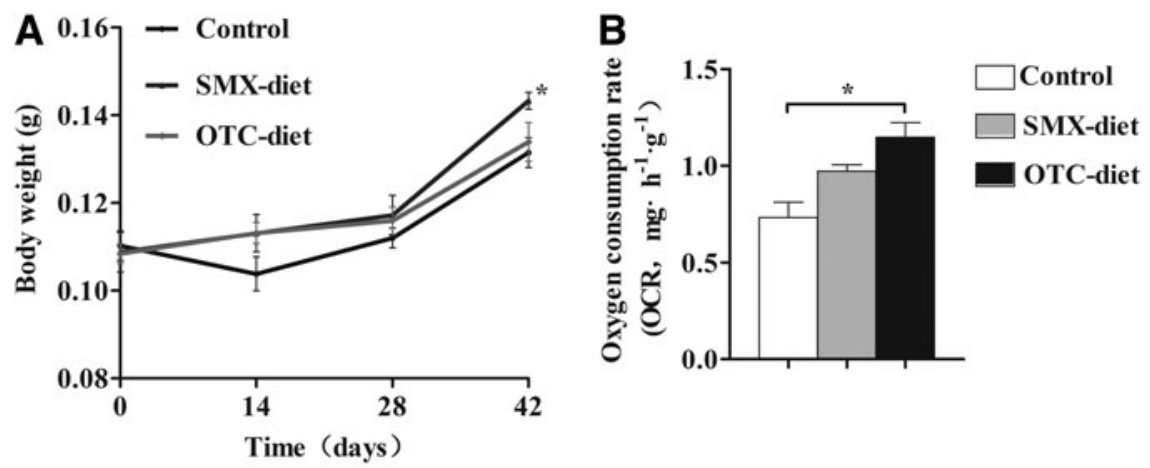

FIG. 1. Influence of feeding SMX and OTC on the body weight and mortality of zebrafish. (A) Growth curve of zebrafish during the 6-week period. (B) Oxygen consumption rate of fish. (C) The mortality of zebrafish challenged with Aeromonas hydrophila. Student's $t$-test was used to detect

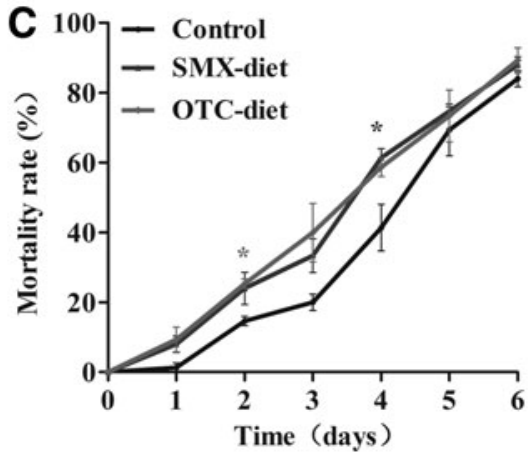
the significant differences between the control and antibiotic exposed groups, $* p<0.05$. OTC, oxytetracycline; SMX, sulfamethoxazole.

examined in control and antibiotic treated groups. The results indicated that the expression level of Glut 2 significantly increased in antibiotic treated groups ( $p<0.05$, Fig. 2D), but the expression level of SGLT1 did not show significant difference between the control and antibiotic treatment (Fig. 2E). The expression level of cationic amino acid transporter, $y^{+} L A T 1$, was higher in antibiotic treated groups, but no significant difference has been detected ( $p>0.05$, Fig. 2F).

\section{Effects of antibiotic exposure on nonspecific immune response in the gut}

The faster killing rate by $A$. hydrophila may be related to impaired immune function and unbalanced inflammation response, so we detected several immune and inflammatory response parameters. ${ }^{28}$ The results indicated that in the intestine, ACP and AKP activities decreased in OTC-diet group $(p<0.05$, Fig. 3A, B). The messenger RNA (mRNA) expression of immune-related pro-inflammatory cytokines $(T N F-\alpha$, $I L-1)$ and the anti-inflammatory cytokine $(T G F-\beta)$ in the gut was also detected, and the results showed that the expression level of $T N F-\alpha$ increased significantly in OTC-diet group $(p<0.05$, Fig. 3C). The relative expression of $I L-1$ was also higher in OTC-diet group, but no significant difference was detected ( $p>0.05$, Fig. 3D). There was no significant difference in the expression level of $T G F-\beta$ between the control and antibiotic treated groups ( $p>0.05$, Fig. 3E).

\section{Oxidative stress response to antibiotic exposure in the gut}

Oxidative stress is often observed in fish suffering from environmental stress. In view of the fact that long-term antibiotic treatment may be one kind of stress, antioxidant response
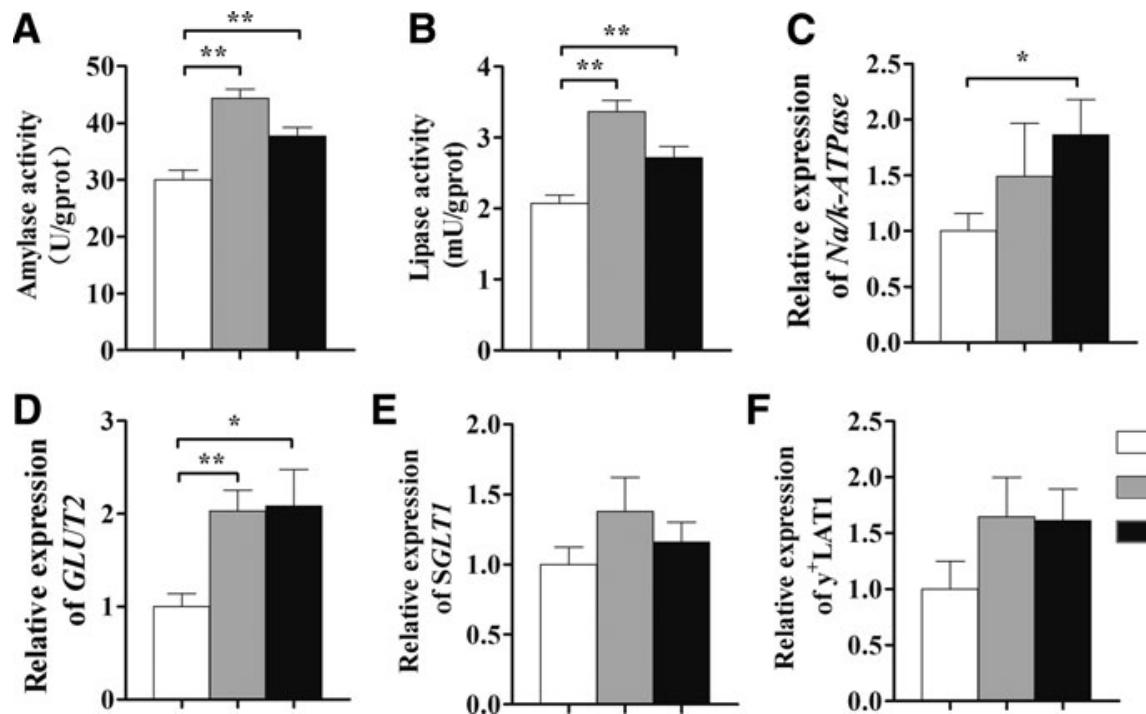

E

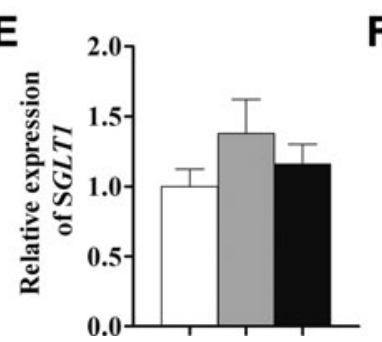

$\mathbf{F}$

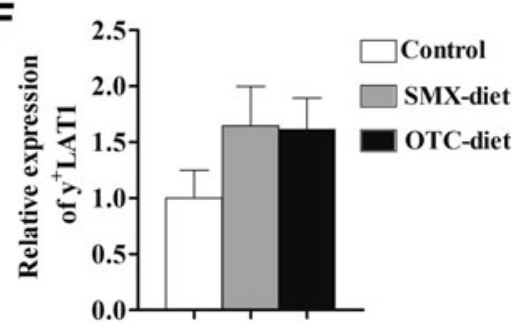

FIG. 2. Influence of feeding antibiotics on the activities of intestinal digestive enzymes and nutrient transporter genes. (A) Amylase activity, (B) lipase activity, (C) $\mathrm{Na}^{+} / \mathrm{K}^{+}$ATPase, (D) Glut2, (E) SGLT1, (F) $y^{+} L A T 1$. Student's $t$-test was used to detect the significant difference between the control and antibiotic exposed groups, $* p<0.05$, $* * p<0.01$. Glut 2 , glucose transporter $2 ; \mathrm{Na}^{+} / \mathrm{K}^{+}$-ATPase, sodium-potassium adenosine triphosphatase; SGLT1, sodium-glucose cotransporter 1; $y^{+} L A T 1$, light chain- $\mathrm{y}^{+} \mathrm{L}$ amino acid transporter- 1 . 
FIG. 3. Influence of feeding antibiotics on the immune-related enzymes and inflammatory factors. (A) AKP activity, (B) ACP activity, (C) $T N F-\alpha$, (D) $I L-1$, and (E) $T G F-\beta$ in zebrafish intestines. Student's $t$-test was used to detect for a significant difference between the control and antibiotic exposed groups, $* p<0.05$. ACP, acid phosphatase; AKP, alkaline phosphatase; $I L-1$, interleukin-1; $T G F-\beta$, transforming growth factor-beta; $T N F-\alpha$, tumor necrosis factor alpha.
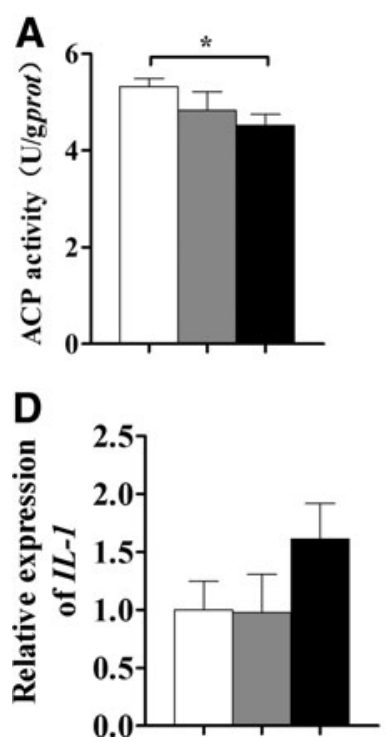
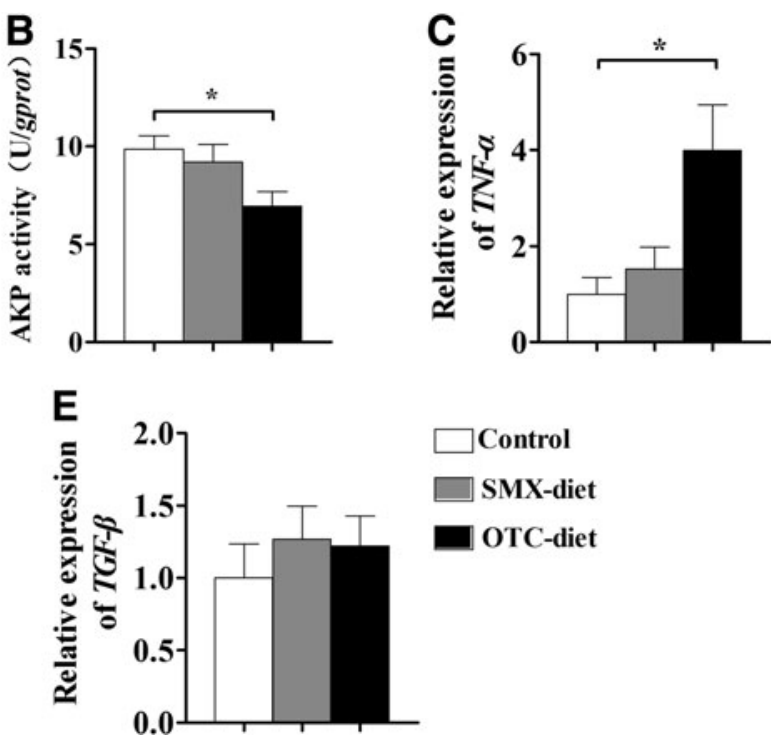

was detected in the intestine. Compared to the control group, MDA content increased significantly $(p<0.05)$ in both SMXdiet and OTC-diet groups (Fig. 4A), while POD activity decreased in these two groups $(p<0.05$, Fig. $4 \mathrm{C})$. Zebrafish in antibiotic treated groups showed lower activities of SOD and GSH compared to the control group, but no significant differences were detected (Fig. 4B, D).

We also detected the activities of MDA, SOD, and POD in liver and muscle, but there were no significant differences between the control and antibiotic treated groups; only $\beta$-oxidation capacity decreased significantly in liver (Supplementary Fig. S1).

\section{Gut microbial communities in zebrafish}

A total of 328,123 high-quality reads were collected by Illumina high-throughput sequencing of barcoded bacterial
$16 \mathrm{~S}$ rRNA genes. The coverage for each sample reached $99 \%$, suggesting good sampling depth. The number of OTUs, the estimators of community richness (ACE and Chao), and diversity (Shannon and Simpson) are shown in Table 1. Results indicated that ACE and Chao indices decreased in OTC-diet group compared to the control group $(p<0.05)$, suggesting that OTC-diet groups had lower bacterial richness compared with the control group. No significant difference has been found in Shannon and Simpson between the control and antibiotic treatment.

The relative abundance $(\%)$ of gut microbiota in each sample at the phylum level is shown in Figure 5A. The dominant phyla in the three groups were Proteobacteria, Firmicutes, Bacteroidetes, Fusobacteria, Planctomycetes, and $\mathrm{CKC} 4$. In the control group, the relative abundances of these six phyla were $51.3 \%, 25.7 \%, 10.9 \%, 6.7 \%, 1.4 \%$, and $2.8 \%$, respectively. In the SMX-diet group, the relative
FIG. 4. Effect of feeding antibiotics on oxidative stress in zebrafish intestine. (A) MDA content, (B) total SOD activity, (C) POD activity, and (D) GSH content. Student's $t$-test was used to detect for significant differences between the control and antibiotic exposed groups, ${ }^{*} p<0.05$. GSH, reduced glutathione; MDA, malondialdehyde; POD, peroxidase; SOD, superoxide dismutase.
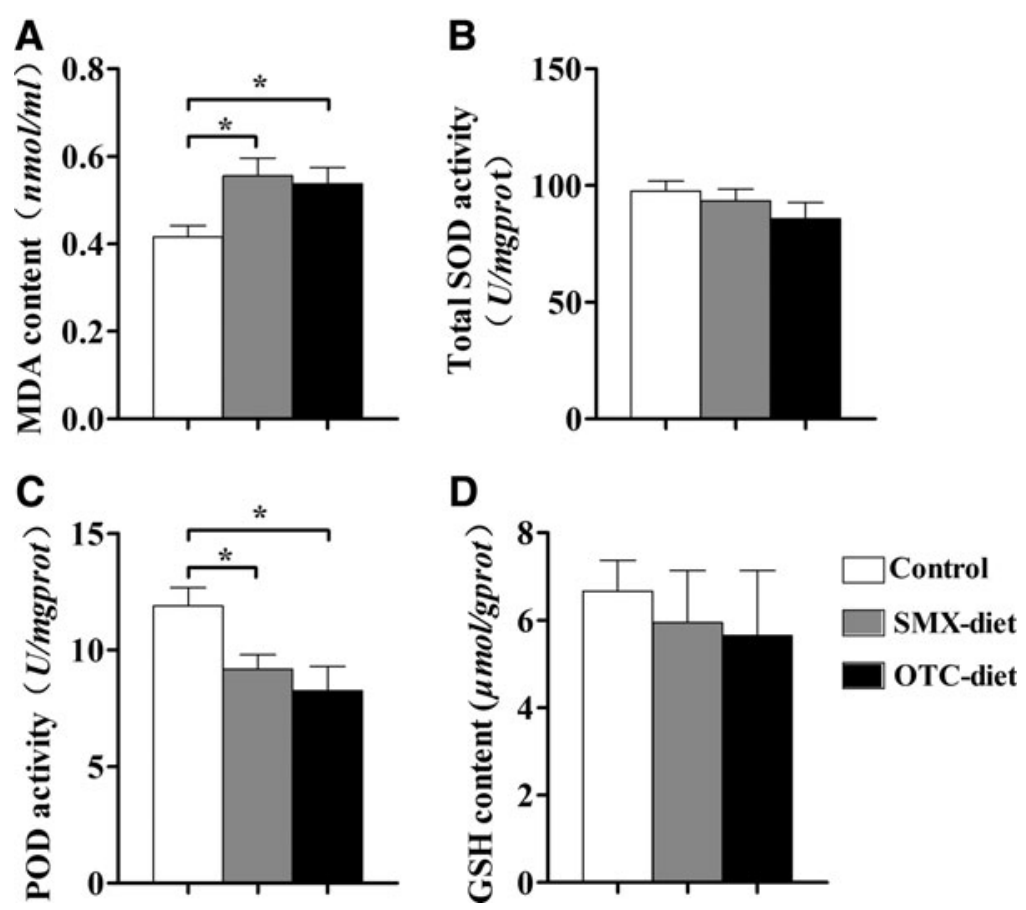
Table 1. Richness and Diversity of Intestinal Microbiota in Zebrafish (Danio Rerio)

\begin{tabular}{lccccccc}
\hline & Reads & OTUs & ACE & Chaol & Shannon & Simpson & Coverage (\%) \\
\hline Control & $38358 \pm 1959$ & $346 \pm 23$ & $364 \pm 24$ & $371 \pm 25$ & $3.35 \pm 0.17$ & $0.09 \pm 0.02$ & $99.90 \pm 0.02$ \\
SMX diet & $36822 \pm 938$ & $273 \pm 40$ & $301 \pm 52$ & $300 \pm 51$ & $3.16 \pm 0.07$ & $0.10 \pm 0.01$ & $99.91 \pm 0.03$ \\
OTC diet & $34248 \pm 3623$ & $218 \pm 19^{\mathrm{a}}$ & $238 \pm 23^{\mathrm{a}}$ & $242 \pm 21^{\mathrm{a}}$ & $3.11 \pm 0.13$ & $0.09 \pm 0.01$ & $99.91 \pm 0.04$ \\
\hline
\end{tabular}

${ }^{\mathrm{a}}$ Represent that compared with control, significant difference $(p<0.05)$.

OTC, oxytetracycline; OTUs, operational taxonomic units; SMX, sulfamethoxazole.
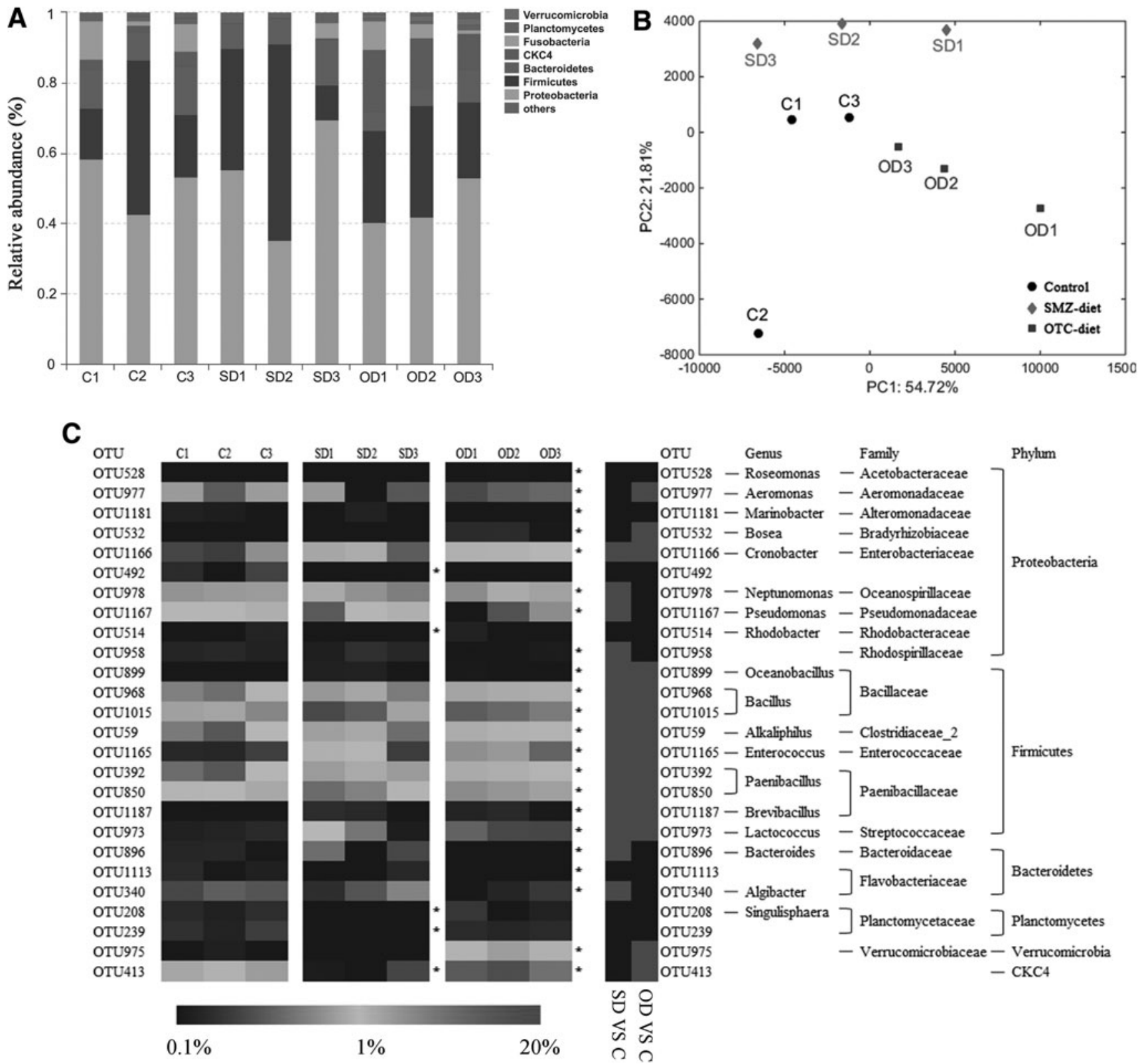

FIG. 5. Effect of feeding antibiotics on the intestinal microbial composition. (A) Gut microbiota composition at the phylum level. Three samples were involved in each group. (B) Principal component analysis of intestinal bacteria in the three groups. (C) Heatmap analysis of selected 26 OTUs. The color bar on the left represents the proportion of each OTU in each sample. The color bar in the middle indicated the comparison of OTU abundance between the antibiotic treated groups and control group. Dark gray indicated that OTU abundance was higher, while black indicated that abundance was lower in antibiotic treated groups than the control ones. *Represents a significant difference compared with the control group based on Student's $t$-test. The affiliation of each OTU was showed on the right panel. C, Control group; OD, OTC diet group; SD, SMX diet group. OTUs, operational taxonomic units. 
Table 2. The percentages of Major Phyla of Gut Microbiota in Three Groups

\begin{tabular}{lccc}
\hline & Control (\%) & SMX diet (\%) & OTC diet $(\%)$ \\
\hline Proteobacteria & $51.3 \pm 8.0$ & $53.4 \pm 17.3$ & $45.1 \pm 6.8$ \\
Firmicutes & $25.7 \pm 16.0$ & $33.4 \pm 23.0$ & $26.4 \pm 5.0$ \\
Bacteroidetes & $10.9 \pm 2.7$ & $9.2 \pm 3.2$ & $6.7 \pm 2.5$ \\
Fusobacteria & $6.7 \pm 4.9$ & $1.5 \pm 2.4$ & $4.4 \pm 3.6$ \\
Planctomycetes & $1.4 \pm 0.2$ & $1.2 \pm 0.7$ & $1.1 \pm 0.4$ \\
CKC4 & $2.8 \pm 1.2$ & $0.2 \pm 0.2^{\mathrm{a}}$ & $13.9 \pm 3.7^{\mathrm{b}}$ \\
\hline
\end{tabular}

${ }^{\mathrm{a}}$ Represents significant difference $(p<0.05)$ compared with Control group.

${ }^{\mathrm{b}}$ Represents significant difference $(p<0.01)$ compared with Control group.

abundances of the six phyla were $53.4 \%, 33.4 \%, 9.2 \%, 1.5 \%$, $1.2 \%$, and $0.2 \%$, respectively. In the OTC-diet group, the relative abundances of those six phyla were $45.1 \%, 26.4 \%$, $6.7 \%, 4.4 \%, 1.1 \%$, and $13.9 \%$, respectively (Table 2). The abundance of CKC4 was significantly influenced by antibiotic treatment.

PCA was used to identify the gut microbial composition in three groups based on the proportion of OTUs, with most of the variations explained by the first two coordinates $54.7 \%$ and $21.8 \%$, respectively (Fig. 5B). SMX-diet group was separated distinctly from the OTC-diet group, but because one sample in control group differed from the other two samples in PC2, no difference was detected between the control and antibiotic treated groups based on the OTU proportion.

Twenty-six OTUs were selected for heatmap analysis according to the standards stated in the methods part (Fig. 5C). Seven OTUs decreased in both antibiotic exposed groups. Furthermore, these OTUs were classified as Proteobacteria, Bacteroidetes, and Planctomycetes. Ten OTUs increased in the antibiotic exposed groups, and nine of them were from Firmicutes.

\section{Discussion and Conclusion}

In aquaculture industry, the effects of dietary antibiotics on weight gain of fish remain inconsistent. For example, sulfonamides were fed to brook trout (Salvelinus fontinalis), brown trout (Salmo trutta), and rainbow trout (Salmo gairdneri), and the results indicated that antibiotics did not show any growth-promoting influence on rainbow trout, while sulfamerazine retarded growth of brook trout. ${ }^{29}$ Channel catfish (Ictalurus punctatus) fed on feeds supplemented with OTC increased the weight gain compared to those in the control. ${ }^{30}$ Increased body weight was also found in Oreochromis niloticus treated with OTC and florfenicol (FLO). ${ }^{31}$ In the present study, SMX treated group showed higher body weight than control group during the experiment, while OTC treatment did not. Due to these inconsistent results, how in-feed antibiotics promote animals' growth is an interesting question.

Based on poultries or pig studies, there are several possible mechanisms underlying this phenomenon. ${ }^{32}$ First, feeding antibiotics inhibit the normal intestinal microbiota, leading to increased nutrient utilization and a reduction in the maintenance costs of the intestinal system. ${ }^{32}$ Second, antibiotics have a nonantibiotic anti-inflammatory effect, which may in turn reduce wasted energy and spare it for production. ${ }^{33}$ Third, antibiotic treatment may permit growth by preventing immunologic stress and associated metabolic changes ${ }^{34}$; and also, growth promotion has been linked to altered condition of the intestinal wall and villus lamina propria, which causes enhanced nutrient digestibility. ${ }^{33,35}$

In the present study, increased digestive enzyme activities have been found in both antibiotic treatments, but no significant difference in immune related enzymes or OCR has been found in SMX group compared with the control group. For OTC treatment, OCR, which is a parameter to examine energy utilization and stress in aquatic animals, ${ }^{36}$ increased. All these results suggested that fish in OTC-treated group need to expand more energy to resistant stress caused by antibiotics instead of growth.

Intestinal immune barrier is an important part of the intestinal health. It has been found that intestinal immune barrier was disturbed by the intestinal inflammatory disorders, such as the upregulation of pro-inflammatory cytokines, decreased anti-inflammatory cytokines in fish, ${ }^{37}$ and impaired immune function. ${ }^{38}$ In our study, we found that longterm dietary OTC treatment upregulated the expression of $T N F-\alpha$, suggesting that long-term OTC treatment triggered the intestinal inflammation. The activity of ACP and AKP, which are important components of the nonspecific immune system, decreased in OTC group.

Numerous studies have showed that antibiotic treatments cause oxidative stress, which also related to the impairment of normal immune functions. ${ }^{12,38,39}$ In the present study, decreased POD and increased MDA content were observed in the gut of zebrafish treated with antibiotics, indicating that long-term antibiotic treatments impair the antioxidative system of zebrafish. All these results were consistent with the fact that killing rate was slightly faster in antibiotic treatment compared with the control group.

Intestinal microbiota plays important roles in protecting the host from pathogen infection. Antibiotic treatment disturbed the intestinal microbiota composition. Although we did not find significant difference between the control and antibiotic treated groups based on PCA, we found that the abundance of some bacteria changed during the antibiotic treatment. It is not surprising that bacteria with endospores were increased in antibiotic treated groups, for example, OTU899, OTU968, and OTU1015 all belong to Bacillaceae, while OTU392 and OTU850 belong to Penibacillaceae, and OTU59 is affiliated to Clostridiaceae.

Furthermore, some bacteria increased in antibiotic treated group. For example, OTU1165 was increased significantly in OTC-diet group and it belongs to Enterococcus, which is one of the important fish pathogens causing severe disease outbreaks in aquaculture facilities. ${ }^{40,41}$ OTU977 was also increased in OTC-diet group and it belongs to Aeromonas, which is the major genera of fish pathogens. ${ }^{42}$ OTU1166 is affiliated to Cronobacter, which is a genus within the family Enterobacteriaceae. Because we got the bacterial composition information based on sequencing, the exact phylogenetic or pathogenic information of these OTUs still need further investigation, but the alteration of the intestinal microbiota may account for the faster killing rate in antibiotic treatment.

In summary, this work evaluated the risk and benefit of long-term dietary antibiotic treatment on zebrafish growth and health. Although oral antibiotics promoted the weight gain of zebrafish to some extent, this benefit was at the expense of lowered immune function and increased killing rate 
when fish were exposed to bacteria challenge. Different antibiotics and concentrations might be the reasons for variations in their effects on fish gut health, but our results suggested that antibiotics with the therapeutic concentration used as growth promoters in fish industry have high risks.

\section{Acknowledgments}

This work was supported by the National Natural Science Foundation of China (grant no. 31672668) and the Science and Technology Commission of Shanghai Municipality (grant no. 16ZR1409900).

\section{Disclosure Statement}

The authors declare that they have no conflict of interest.

\section{References}

1. FAO: The State of World Fisheries and Aquaculture 2014. FAO, Rome, 2014.

2. Mo WY, Chen ZT, Leung HM, Leung AOW. Application of veterinary antibiotics in China's aquaculture industry and their potential human health risks. Environ Sci Pollut Res Int 2017;24:8978-8989.

3. Kollner B, Wasserrab B, Kotterba G, Fischer U. Evaluation of immune functions of rainbow trout (Oncorhynchus mykiss)-how can environmental influences be detected? Toxicol Lett 2002;131:83-95.

4. Montero D, Izquierdo MS, Tort L, Robaina L, Vergara JM. High stocking density produces crowding stress altering some physiological and biochemical parameters in gilthead seabream, Sparus aurata, juveniles. Fish Physiol Biochem 1999;20:53-60.

5. Wei Q: Social and economic impacts of aquatic animal health problems in aquaculture in China. In: Primary Aquatic Animal Health Care in Rural, Small-Scale, Aquaculture Development. Arthur JR, Phillips MJ, Subasinghe RP, Reantaso MB, and MacRae IH (eds), pp. 55-61, FAO Fisheries Technical Paper No. 406, FAO, Rome, 2002.

6. Xu WH, Zhu XB, Wang XT, Deng LP, Zhang G. Residues of enrofloxacin, furazolidone and their metabolites in Nile tilapia (Oreochromis niloticus). Aquaculture 2006;254:1-8.

7. Carvalho IT, Santos L. Antibiotics in the aquatic environments: a review of the European scenario. Environ Int 2016;94:736-757.

8. Currie J, Lin WC, Zhang W. Patient knowledge and antibiotic abuse: evidence from an audit study in China. J Health Econ 2011;30:933-949.

9. He S, Zhou Z, Liu Y, Cao Y, Meng K, Shi P, et al. Effects of the antibiotic growth promoters flavomycin and florfenicol on the autochthonous intestinal microbiota of hybrid tilapia (Oreochromis niloticus female symbol $\times$ O. aureus male symbol). Arch Microbiol 2010;192:985-994.

10. Sarmah AK, Meyer MT, Boxall ABA. A global perspective on the use, sales, exposure pathways, occurrence, fate and effects of veterinary antibiotics (VAs) in the environment. Chemosphere 2006;65:725-759.

11. Seyfried EE, Newton RJ, Rubert KF, Pedersen JA, McMahon KD. Occurrence of tetracycline resistance genes in aquaculture facilities with varying use of oxytetracycline. Microb Ecol 2010;59:799-807.

12. Yonar ME, Yonar SM, Silici S. Protective effect of propolis against oxidative stress and immunosuppression induced by oxytetracycline in rainbow trout (Oncorhynchus mykiss, W.). Fish Shellfish Immun 2011;31:318-325.

13. Nakayama T, Hoa TTT, Harada K, Warisaya M, Asayama M, Hinenoya A, et al. Water metagenomic analysis reveals low bacterial diversity and the presence of antimicrobial residues and resistance genes in a river containing wastewater from backyard aquacultures in the Mekong Delta, Vietnam. Environ Pollut 2017;222:294-306.

14. Chen H, Liu S, Xu XR, Zhou GJ, Liu SS, Yue WZ, et al. Antibiotics in the coastal environment of the Hailing Bay region, South China Sea: spatial distribution, source analysis and ecological risks. Mar Pollut Bull 2015;95:365-373.

15. Nguyen Dang Giang C, Sebesvari Z, Renaud F, Rosendahl I, Hoang Minh Q, Amelung W. Occurrence and dissipation of the antibiotics sulfamethoxazole, sulfadiazine, trimethoprim, and enrofloxacin in the Mekong Delta, Vietnam. PLoS One 2015;10:e0131855.

16. Niklasson L, Sundh H, Fridell F, Taranger GL, Sundell K. Disturbance of the intestinal mucosal immune system of farmed Atlantic salmon (Salmo salar), in response to longterm hypoxic conditions. Fish Shellfish Immunol 2011;31: 1072-1080.

17. Doherty MM, Charman WN. The mucosa of the small intestine-how clinically relevant as an organ of drug metabolism? Clin Pharmacokinet 2002;41:235-253.

18. Choi JJ, Eum SY, Rampersaud E, Daunert S, Abreu MT, Toborek M. Exercise attenuates PCB-induced changes in the mouse gut microbiome. Environ Health Perspect 2013; 121:725-730.

19. Atarashi K, Tanoue T, Shima T, Imaoka A, Kuwahara T, Momose Y, et al. Induction of colonic regulatory $\mathrm{T}$ cells by indigenous Clostridium species. Science 2011;331:337-341.

20. Rodrigues S, Antunes SC, Correia AT, Nunes B. Rainbow trout (Oncorhynchus mykiss) pro-oxidant and genotoxic responses following acute and chronic exposure to the antibiotic oxytetracycline. Ecotoxicology 2017;26:104-117.

21. Zhang ML, Sun YH, Chen K, Yu N, Zhou ZG, Chen LQ, et al. Characterization of the intestinal microbiota in Pacific white shrimp, Litopenaeus vannamei, fed diets with different lipid sources. Aquaculture 2014;434:449-455.

22. Trovó AG, Nogueira RF, Agüera A, Fernandez-Alba AR, Sirtori C, Malato S. Degradation of sulfamethoxazole in water by solar photo-fenton. Chemical and toxicological evaluation. Water Res 2009;43:3922-3931.

23. Rigos G, Troisi GM. Antibacterial agents in Mediterranean finfish farming: a synopsis of drug pharmacokinetics in important euryhaline fish species and possible environmental implications. Rev Fish Biol Fisheries 2005;15: 53-73.

24. China MoAotPsRo. NY 5071-2002, guidelines for the use of non-polluted food and fishery drugs. Ocean Fishery 2007;12:39-41.

25. Schnappinger D, Hillen W. Tetracyclines: antibiotic action, uptake, and resistance mechanisms. Arch Microbiol 1996; 165:359-369.

26. Yonar ME. The effect of lycopene on oxytetracyclineinduced oxidative stress and immunosuppression in rainbow trout (Oncorhynchus mykiss, W.). Fish Shellfish Immun 2012;32:994-1001.

27. Elia AC, Ciccotelli V, Pacini N, Doerr AJM, Gili M, Natali $\mathrm{M}$, et al. Transferability of oxytetracycline (OTC) from feed to carp muscle and evaluation of the antibiotic effects on antioxidant systems in liver and kidney. Fish Physiol Biochem 2014;40:1055-1068. 
28. Zhou L, Limbu SM, Shen ML, Zhai WY, Qiao F, He A, et al. Environmental concentrations of antibiotics impair zebrafish gut health. Environ Pollut 2018;235:245-254.

29. Snieszko SF, Wood EM. The effect of some sulfonamides on the growth of brook trout, brown trout, and rainbow trout. Trans Am Fish Soc 1955;84:86-92.

30. Sanchez-Martinez JG, Perez-Castaneda R, Rabago-Castro JL, Aguirre-Guzman G, Vazquez-Sauceda ML. A preliminary study on the effects on growth, condition, and feeding indexes in channel catfish, Ictalurus punctatus, after the prophylactic use of potassium permanganate and oxytetracycline. J World Aquaculture Soc 2008;39: 664-670.

31. Reda RM, Ibrahim RE, Ahmed E-N, El-Bouhy ZM. Effect of oxytetracycline and florfenicol as growth promoters on the health status of cultured Oreochromis niloticus. Egypt J Aquat Res 2013;39:241-248.

32. Gaskins HR, Collier CT, Anderson DB. Antibiotics as growth promotants: mode of action. Anim Biotechnol 2002; 13:29-42.

33. Niewold TA. The nonantibiotic anti-inflammatory effect of antimicrobial growth promoters, the real mode of action? A hypothesis. Poultry Sci 2007;86:605-609.

34. Roura E, Homedes J, Klasing KC. Prevention of immunologic stress contributes to the growth-permitting ability of dietary antibiotics in chicks. J Nutr 1992;122:2383-2390.

35. Jukes HG, Hill DC, Branion HD. Effect of feeding antibiotics on the intestinal tract of the chick. Poultry Sci 1956; 35:716-723.

36. Meade ME, Doeller JE, Kraus D, Watts SA. Effects of temperature and salinity on weight gain, oxygen consumption rate, and growth efficiency in juvenile red-claw crayfish Cherax quadricarinatus. J World Aquaculture Soc 2010;33:188-198.

37. Wu P, Jiang WD, Jiang J, Zhao J, Liu Y, Zhang YA, et al. Dietary choline deficiency and excess induced intestinal inflammation and alteration of intestinal tight junction protein transcription potentially by modulating NF-kappaB, STAT and p38 MAPK signaling molecules in juvenile Jian carp. Fish Shellfish Immunol 2016;58:462-473.
38. Pan H, Li LY, Li JM, Wang WL, Limbu SM, Degrace P, et al. Inhibited fatty acid beta-oxidation impairs stress resistance ability in Nile tilapia (Oreochromis niloticus). Fish Shellfish Immunol 2017;68:500-508.

39. Alak G, Yeltekin AC, Tas IH, Ucar A, Parlak V, Topal A, et al. Investigation of 8-OHdG, CYP1A, HSP70 and transcriptional analyses of antioxidant defence system in liver tissues of rainbow trout exposed to eprinomectin. Fish Shellfish Immun 2017;65:136-144.

40. Martins ML, Mourino JLP, Amaral GV, Vieira FN, Dotta $\mathrm{G}$, Jatoba AMB, et al. Haematological changes in Nile tilapia experimentally infected with Enterococcus sp. Braz J Biol 2008;68:657-661.

41. Rahman M, Rahman MM, Deb SC, Alam MS, Alam MJ, Islam MT. Molecular identification of multiple antibiotic resistant fish pathogenic Enterococcus faecalis and their control by medicinal herbs. Sci Rep 2017;7:1-11.

42. Plumb JA: Infectious diseases of tilapia. In: Tilapia Aquaculture in the Americas. Costapierce BA and Rakocy JE (eds), pp. 212-222, World Aquaculture Society, Baton Rouge, LA, 1997.

Address correspondence to:

Meiling Zhang, PhD

School of Life Sciences

East China Normal University

500 Dong Chuan Road

Shanghai 200241

China

E-mail: mlzhang@bio.ecnu.edu.cn

Zhen-Yu Du, PhD

School of Life Sciences

East China Normal University

500 Dong Chuan Road

Shanghai 200241

China

E-mail: zydu@bio.ecnu.edu.cn 